\title{
Larval development of Euphausia pacifica in the laboratory: variability in developmental pathways
}

\author{
Leah R. Feinberg ${ }^{1, *}$, C. Tracy Shaw ${ }^{1}$, William T. Peterson ${ }^{2}$ \\ ${ }^{1}$ Cooperative Institute for Marine Resources Studies, Hatfield Marine Science Center, 2030 South Marine Science Drive, \\ Newport, Oregon 97356, USA \\ ${ }^{2}$ NMFS, Hatfield Marine Science Center, 2030 South Marine Science Drive, Newport, Oregon 97356, USA
}

\begin{abstract}
Gravid adult female Euphausia pacifica were collected off Newport, Oregon, USA and transferred to the laboratory, where females spawned eggs, eggs hatched, and larvae were reared at $10.5^{\circ} \mathrm{C}$. We fed 4 cohorts of larvae to excess with a combination of phytoplankton species and monitored them daily until they reached the juvenile stage. The euphausiids were maintained separately (1 individual per jar) from the third furcilia (FIII) to the juvenile stage to observe developmental pathways. Individual cohorts developed at nearly the same rate until the first furcilia stage, after which 2 cohorts began to develop significantly faster than the others. Median time to the juvenile stage ranged from 51.9 to $60.6 \mathrm{~d}$, with significant differences among cohorts. The first calyptopis stage and the third furcilia stage lasted longer than any other stages and appear to be bottlenecks in the development of this species. Individual development from FIII to juvenile varied widely both within and among cohorts. We observed 4 main developmental pathways. Over half of the euphausiids skipped 1 development stage between FIII and juvenile (58\%), and none skipped multiple stages. There was no tendency for individuals from the same cohort to follow the same developmental pathway. This variability in development may be even higher in the field and could impact mortality calculations and cohort analysis from field samples.
\end{abstract}

KEY WORDS: Euphausia pacifica $\cdot$ Larval development time $\cdot$ Developmental pathways

\section{INTRODUCTION}

Euphausia pacifica is 1 of the 2 dominant euphausiid species found on the Oregon shelf (Brinton 1962, Mauchline \& Fisher 1969). E. pacifica is abundant throughout much of the North Pacific, ranging from Mexico northward in the California Current, westward around to the Sea of Japan and southward to the East China Sea (Brinton 1962). This species is highly successful in the open ocean, in dynamic shelf systems, and in protected inland basins (Bollens et al. 1992). Regional differences can exist among populations. For instance, timing of spawning is variable, occurring nearly year-round off southern California (Brinton 1976), March through October off Oregon (Smiles \& Pearcy 1971, Feinberg \& Peterson 2003), April through May in Puget Sound (Ross et al. 1982), May through June in the North Pacific (Ponomareva 1966) and February through May in Toyama Bay, Japan (Iguchi et al. 1993). Estimated growth rates and life expectancies also vary extensively among regions (Siegel \& Nicol 2000).

In this study we monitored the development of Euphausia pacifica from egg-hatching to the juvenile stage using individuals from the Oregon upwelling region. A similar study of E. pacifica development was carried out by Ross (1981) with individuals from Puget Sound, Washington. We repeated many aspects of Ross' (1981) study because life-history differences, such as spawning season and patterns, exist between these populations (Feinberg \& Peterson 2003). Differences in life-history and environmental conditions between these study areas suggested that we should not assume that results from a Puget Sound population (which is isolated from the Pacific Ocean) are applicable to the population in our study area. Differences between our methodology and that of Ross (1981) 
included our use of a diet consisting of a mix of algal species and of an experimental temperature typical of Oregon coastal waters.

The development of Euphausia pacifica includes 2 naupliar stages, a metanauplius stage, 3 calyptopis stages and 5 to 7 furcilia stages (Boden 1950, Suh et al. 1993). Variability in developmental pathways has been reported previously (Boden 1950, Ross 1981, Knight 1984, Rumsey 1999). Most studies only refer to variability in the developmental pathway for the first 2 furcilia stages (Boden 1950, Knight 1984, Rumsey 1999), because variability in development of these stages can be determined from preserved samples by counting and staging the pleopods (Knight 1984). Variability in developmental pathway through the later furcilia stages cannot be determined from preserved samples because more than 1 instar is possible within a given stage and instars within a stage are morphologically identical. Thus, it is impossible to tell whether the later furcilia stages (FIII to FVII) of a field-collected individual had one or multiple instars within the same development stage or skipped a stage. To document variability in the developmental patterns of later furcilia stages, we maintained the euphausiids individually in jars from FIII to the juvenile stage, and observed them daily during development experiments in the laboratory.

This study contributes to a larger study of the population dynamics and vital rates of euphausiid populations in the Oregon upwelling zone conducted as part of the US GLOBEC program. As with other similar euphausiid studies, a large portion of our study of population dynamics is focused on the collection of euphausiids from the field at frequent intervals (Smiles \& Pearcy 1971, Brinton 1976, Ross et al. 1982, Tanasichuk 1998). Such field data can be used to estimate in situ growth, productivity and mortality rates, but before we can estimate these rates we must have accurate information about time to stage (i.e. time to attain each stage), stage duration and developmental pathways. The larval development experiments discussed in this paper were designed to help in the interpretation of field data as well as determine the applicability of Ross' (1981) results to oceanic populations of Euphausia pacifica. This paper reports on our findings for the time to stage and stage duration for cohorts and individual variability in development pathways for this population of E. pacifica.

\section{MATERIALS AND METHODS}

Experimental conditions. We collected adult $E \mathrm{E}$ phausia pacifica at a station $\left(44^{\circ} 40^{\prime} \mathrm{N}, 124^{\circ} 40^{\prime} \mathrm{W}\right.$; $300 \mathrm{~m}$ depth) $25 \mathrm{n}$ miles off Newport, Oregon in July
2001. The euphausiids were collected at night, using obliquely towed, $60 \mathrm{~cm}$ diameter, $202 \mu \mathrm{m}$-mesh bongo nets with solid cod ends. The euphausiids were kept cool and in the dark and transported (approximately $3 \mathrm{~h}$ ) to a shore-based cold room set at $10.5^{\circ} \mathrm{C}$. This temperature is typical for upper water-column conditions off the coast of Newport (Feinberg \& Peterson 2003). Gravid females, identified by the presence of purple-colored ovaries, were sorted and placed individually in 11 jars of filtered seawater and monitored throughout the night until they spawned. The eggs were placed in shallow dishes and checked every $4 \mathrm{~h}$ under a dissecting microscope until they hatched. After hatching, we selected 4 cohorts of nauplii (a cohort represents a batch of eggs spawned from a single female) for our experiments. Larvae were kept in clear, round containers with densities decreasing as stage advanced, using the densities suggested by Ross (1981). Container size started at $150 \mathrm{ml}$ and increased to $500 \mathrm{ml}$ by the time FIIIs were maintained individually. Experiments were maintained in the dark, with brief exposure to light while being staged, counted and fed each day.

Larvae were staged with the aid of a dissecting microscope using the descriptions of Boden (1950), and were transferred daily by pipette to clean water. Once they reached the first calyptopis stage (the first feeding stage), they were fed to excess ( $>100 \mu \mathrm{g}$ carbon $\mathrm{l}^{-1}$ ). Initially, the euphausiids were fed a mixture of Isochrysis sp. (flagellate) and Chaetoceros calcitrans (diatom). As they grew larger, Rhodomonas salina (flagellate) and Heterocapsa triquetra (dinoflagellate) were added to the diet and food concentrations were gradually increased. All individuals were fed from the same beaker of mixed algae to insure a consistent feeding environment among culture containers. Disappearance of algae was not measured directly, but we observed color in the water at every daily check, and full guts of the euphausiids, suggesting that there was not a period of low food concentration between feedings. Algae were grown in batch cultures under constant light, at $16^{\circ} \mathrm{C}$, using a culture medium described by Matthiessen \& Toner (1966). Once the euphausiids reached the third furcilia stage (FIII), they became active swimmers, making it impossible to examine them closely enough to determine stage. However, we were able to follow the developmental pathways of individuals by examining the molts. Therefore, beginning with FIII, the euphausiids were maintained individually in $500 \mathrm{ml}$ jars and checked daily for molts. Molts were staged with the aid of a dissecting microscope. Occasionally, molts could not be staged due to damaged or missing parts. Only individuals with a complete molt history were used for individual analyses of developmental path- 
way. The water was changed and the euphausiids fed to excess twice a week from FIII to juvenile stage. The fourth and fifth furcilia stages (FIV and FV) were treated as 1 stage, in part because this stage could not be distinguished by examining the molt, and in part because FV is a stage that is rarely collected in the field, suggesting it is often skipped in the development of this species in the NE Pacific (Ross 1981, L. R. Feinberg et al. pers. obs. from Oregon field samples). The experiment ended when all euphausiids had reached the juvenile stage (they were maintained beyond this point for lipofuscin calibration and egg-production experiments described elsewhere).

Analysis of data. We recorded the time of hatching and the time when the euphausiids were checked each day. This enabled us to keep track of development time from hatching in fractions of days. Time ' 0 ' was the median time of egg-hatching for each cohort. We computed cumulative percentages of individuals which were in, or had passed through, a given stage and plotted them versus time. We fitted the arcsinetransformed, cumulative frequency curves with regression lines (Sokal \& Rohlf 1969) and used these lines to determine the median time to stage, defined as the time at which $50 \%$ of the individuals were either in, or had molted out of, a development stage; stage duration was calculated as the time between median development times (Landry 1983, Uye 1988). Cohort 4 was omitted from calculations of the overall experimental medians because of its exceptionally high mortality; however, it was included in all comparisons between cohorts. In many cases there were a few individuals that lagged behind in a given stage and took much longer to progress than the others. Before regression analysis, we truncated the tails in order to keep the stragglers from biasing the slopes of the

Table 1. Euphausia pacifica. Hatching and survival of the different cohorts, showing female total length (TL) and brood size (no. of eggs). $\mathrm{S}_{\mathrm{e}-\mathrm{j}}$ : \% survival from egg to juvenile; $\mathrm{S}_{\mathrm{N} 1-\mathrm{j}}$ : $\%$ survival from N1 to juvenile

\begin{tabular}{|lccccc|}
\hline Cohort & $\begin{array}{c}\mathrm{TL} \\
(\mathrm{mm})\end{array}$ & $\begin{array}{c}\text { Brood } \\
\text { size }\end{array}$ & $\begin{array}{c}\% \\
\text { hatching }\end{array}$ & $\mathrm{S}_{\mathrm{e}-\mathrm{j}}$ & $\mathrm{S}_{\mathrm{N} 1-\mathrm{j}}$ \\
\hline 1 & 21.5 & 190 & 86 & 12 & 13.5 \\
2 & 22.5 & 321 & 86 & 12 & 15.2 \\
3 & 22.8 & 321 & 94 & 12 & 12.6 \\
4 & 21.1 & 228 & 50 & 4 & 6 \\
\hline
\end{tabular}

regression lines. The slopes of the development curves for all cohorts were compared using ANCOVA analysis, and individual cohort pairs were compared using Tukey's HSD test (Zar 1999). Survival was calculated as the proportion of euphausiids that lived to the juvenile stage, relative to the number of eggs produced in a batch and to the number of first naupliar stage (N1) hatched.

\section{RESULTS}

\section{Hatching and survival}

The total lengths of spawning females used in this experiment varied by $<2 \mathrm{~mm}$ (Table 1). Brood sizes ranged from 190 to 321 eggs. Egg-hatching was 86\% for Cohorts 1 and 2, $94 \%$ for Cohort 3 and $50 \%$ for Cohort 4 (Table 1). The average time to hatching was 39 h. The fourth cohort had significantly lower survivorship than the other 3 , especially from the egg to the juvenile stage (Table 1, Fig. 1). All cohorts experienced a high degree of mortality in the transition through the first calyptopis stage (approximately 6 to $12 \mathrm{~d}$ after hatching). Nearly half of the euphausiids had died by this point. There was very little mortality from the third furcilia stage through to juvenile (after approximately 30 d, Fig. 1). On average, $92 \%$ of all mortality between the N1 and juvenile stages occurred before the third furcilia stage. Although our experiment ended at this point, many of the juveniles survived to adulthood and lived for up to $17 \mathrm{mo}$ in the laboratory before being sacrificed for other experiments. 


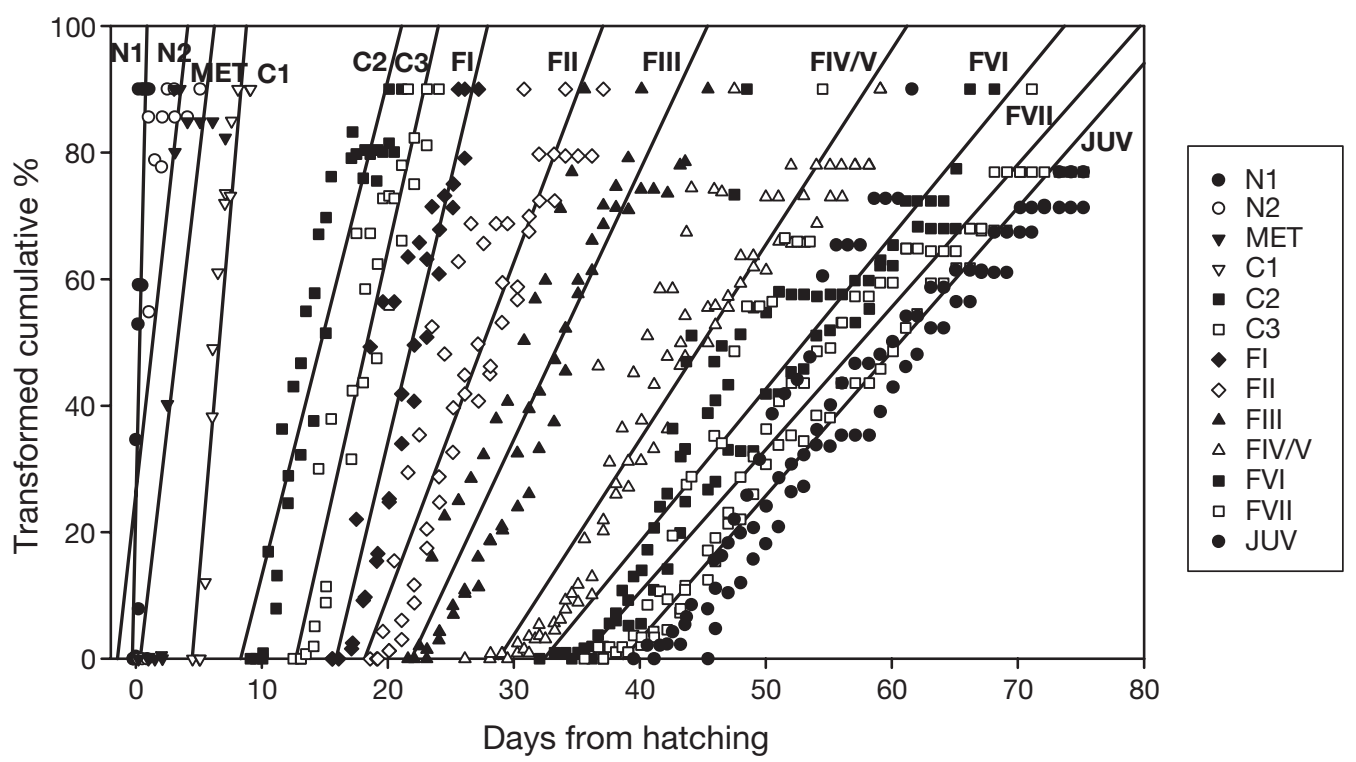

Fig. 2. Euphausia pacifica. Cumulative percent (arcsine-transformed) of individuals that passed into or through each specified stage on a given day of the experiment for Cohorts 1 to 3 combined. Lines represent linear regressions fitted to transformed data

\section{Larval development}

The declining slopes of successive regression lines fitted to the cumulative percent of individuals in each stage vs. time indicate increasing development time with increasing stage (Fig. 2). Overall variability in time to stage and time in each stage also increased for individuals with advancing development stage. This increased variability among development of individuals led to decreased slopes for the resulting regression lines, especially in the furcilia stages. Estimates of median development times are shown in Table 2 . Median time to the juvenile stage was $58.4 \mathrm{~d}$ (Table 2).

Table 2. Euphausia pacifica. Median time (d) to attain the various stages and range in stage for Cohorts 1 to 3 combined. Range in stage shown as range in experiment days (total days). MET: metanauplius; JUV: juvenile

\begin{tabular}{|lcc|}
\hline Stage & $\begin{array}{c}\text { Median } \\
\text { time }\end{array}$ & $\begin{array}{c}\text { Range in } \\
\text { stage }\end{array}$ \\
\hline N1 & 0.3 & $0-4.0(4)$ \\
N2 & 1.1 & $1.0-7.1(6.1)$ \\
MET & 3.0 & $1.0-7.5(6.5)$ \\
C1 & 6.4 & $5.5-20.6(15.1)$ \\
C2 & 14.1 & $10.1-23.1(13)$ \\
C3 & 17.8 & $13.5-26.1(12.6)$ \\
FI & 21.3 & $17.1-36.2(19.1)$ \\
FII & 26.7 & $19.6-43.6(24)$ \\
FII & 32.4 & $22.5-58.2(35.7)$ \\
FIV/V & 43.4 & $27.1-67.1(40)$ \\
FVI & 51.0 & $33.2-75.2(42)$ \\
FVII & 55.4 & $36.7-75.0(38.3)$ \\
JUV & 58.4 & 40.6 \\
\hline
\end{tabular}

Range in stage, defined as the time between the day the first individual of a stage appeared and the day the last individual remained in that stage, increased greatly at Stage FIII to a mean of approximately $36 \mathrm{~d}$ and remained at that approximate level during subsequent stages (Table 2).

Stage duration increased with increasing development stage up to FIII, and then declined (Fig. 3). There were 2 stages, Calyptopis 1 (C1) and FIII, which were by far the longest stages in this experiment ( 7.7 and $11 \mathrm{~d}$ respectively). In our experiments, there were often 2 to 3 instars within the third furcilia stage. We were unable to tell whether there were multiple instars in the other prolonged stage, $\mathrm{C} 1$, since the euphausiids were not isolated at that point in the experiment and molts could not be traced to specific individuals.

\section{Variability in development among cohorts}

All 4 cohorts followed a similar pattern of development (Fig. 4); 3 segments of development (egg to C1, C1 to FIII, FIII to juvenile) were noticeably delineated by the 'bottleneck' C1 and FIII stages. The median development times and patterns of Cohorts 2 and 3 were quite similar, but despite development under exactly the same experimental conditions, Cohorts 1 and 4 reached the median times to the various stages consistently faster (Fig. 4). This trend was especially noticeable throughout the furcilia stages. By the time Cohorts 1 and 4 had reached FII, they were approximately $3 \mathrm{~d}$ ahead of the median development times of Cohorts 2 and 3 . This gap continued to widen until 


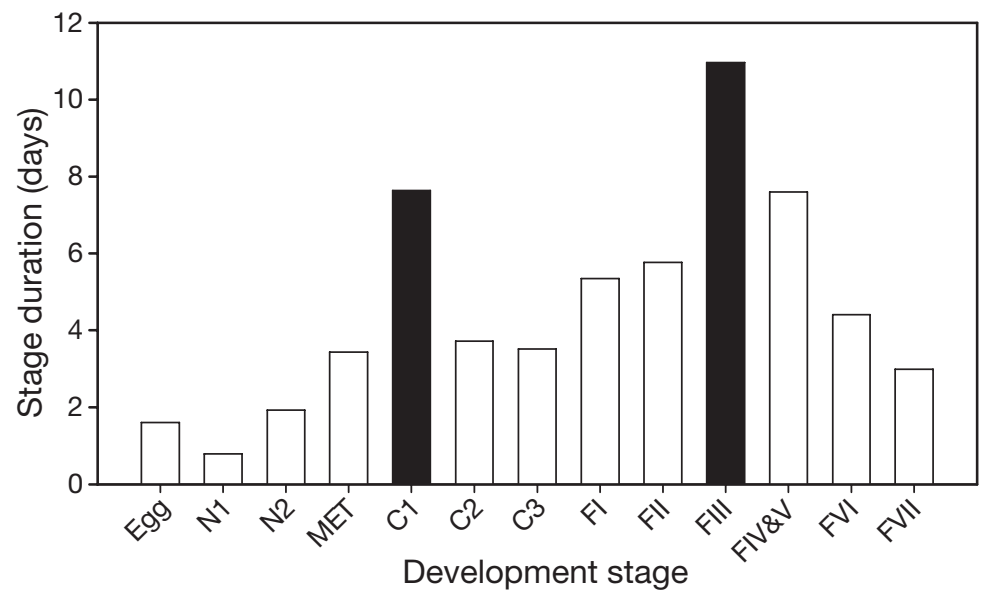

Fig. 3. Euphausia pacifica. Development stage duration. Time between median development times for each stage (Cohorts 1 to 3 combined). Black columns show bottleneck stages (C1 and FIII)

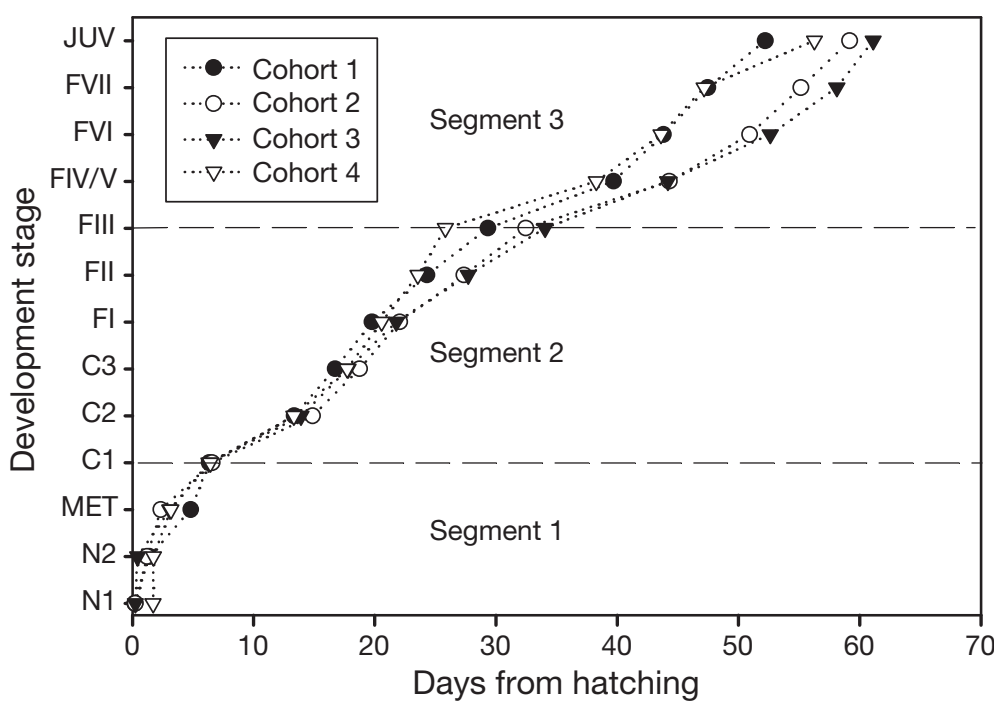

Fig. 4. Euphausia pacifica. Median time to each development stage for 4 cohorts. Development is broken into 3 stage-segments. Segment 1: Stages N1 to C1; Segment 2: Stages C1 to FIII; Segment 3: Stages FIII to juvenile

Furcilia VII (FVII), by which time Cohorts 1 and 4 were approximately 8 to $10 \mathrm{~d}$ and nearly 2 stages ahead of the other 2 cohorts. This faster development pace allowed Cohort 1 to reach juvenile stage 7 , and $9 \mathrm{~d}$ earlier than Cohorts 2 and 3 respectively. However, Cohort 4 was slow to complete the final molt to juvenile, and its median development time to the juvenile stage was $58 \mathrm{~d}$, far closer to that of Cohorts 2 and 3 than to the $52 \mathrm{~d}$ calculated for Cohort 1 (Fig. 4).

Comparison of individual development segments revealed no significant difference in slope between cohorts for the first 2 segments (N1 to $\mathrm{C} 1$ and $\mathrm{C} 1$ to FIII). However, the ANCOVA analysis comparing all cohorts in the third segment (FIII to juvenile) showed a marginal differ- ence at the $p=0.06$ significance level. Analysis of the third-segment slopes of individual cohort pairs using Tukey's HSD test showed a significant difference between Cohorts 1 and 3 and Cohorts 3 and $4(\mathrm{p}<0.05)$ and somewhat less significant differences between Cohorts 1 and 2 and Cohorts 2 and $4(\mathrm{p}<0.10)$. The lag in development for Cohort 4 at the end of the experiment may have been influenced by the high level of mortality in that cohort, of which only 9 individuals survived to the juvenile stage.

\section{Individual developmental pathways}

Individual development was followed from the time euphausiids reached FIII until they became juveniles. Using only those individuals with complete molt records ( $\mathrm{n}=67$ ), we found that individuals followed one of 4 main developmental pathways (Fig. 5): Pathway 1 contained $42 \%$ of the euphausiids, followed by Pathway 2 (33\%), Pathway $3(19 \%)$ and Pathway $4(6 \%)$. More individuals skipped a stage (Pathways 2 to $4=58 \%$ ) than passed through all stages (Pathway 1,42\%). The later development stages were more likely to be skipped (FVII $=33 \%$ vs. FIV $/ \mathrm{V}=6 \%$ ). No individual skipped more than 1 developmental stage.

The number of instars per furcilia stage varied between individuals. A single instar was most common for all stages after FIII, however, at FIII, over $90 \%$ of the euphausiids had more than 1 instar. Up to 4 instars were observed at stages FIII and FVII, and up to 3 instars at FIV/V and FVI (Fig. 6). The percentage of multiple instars per stage generally decreased with increasing development stage. At FIV/V, 65\% of the euphausiids had 1 instar, $31 \%$ had 2 instars, and only $3 \%$ had 3 instars. We observed a single instar for FVI and FVII in $80 \%$ and $75 \%$ of the euphausiids, respectively. Pathway 1 (no stages skipped) had 5 to 11 instars between the FIII and juvenile stages (Fig. 7). In contrast, individuals that followed the other 3 pathways (whereby the euphausiids skipped 1 stage) had a range of 4 to 7 instars during this period (Fig. 7). Average intermolt period was similar for each pathway $(\sim 3 \mathrm{~d})$, but the number of instars per pathway differed, resulting in divergent development times from the FIII to juvenile stage. Individuals that did not skip any stages had an average development time from FIII to juvenile of $27.1 \mathrm{~d}$, while those that skipped a stage developed in an average of $20.5 \mathrm{~d}$. Euphausiids that skipped a stage were most likely to skip FVII $(63 \%)$. 


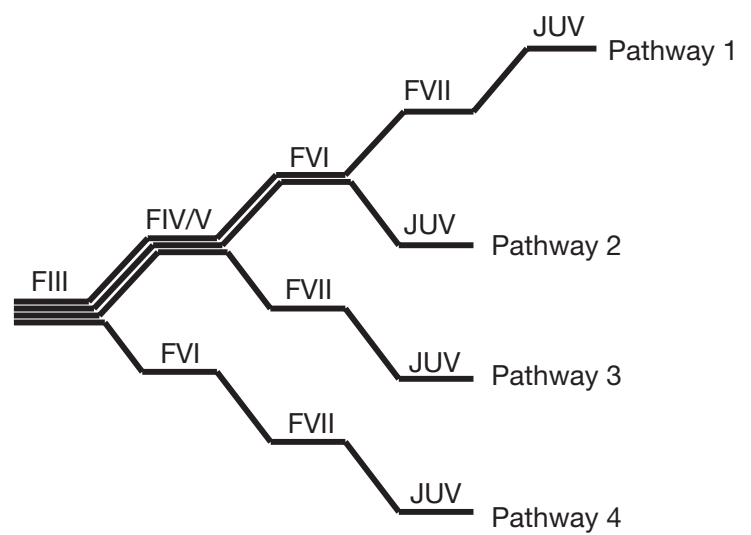

Fig. 5. Euphausia pacifica. Developmental pathways followed by furcilia in this study. JUV: juvenile

There was no tendency for individuals from the same cohort to follow the same developmental pathway (Table 3). However, the breakdown in percentage of individuals following certain pathways showed some patterns. In Cohort 1, equal numbers of euphausiids followed Pathways 1 and 2. The most common pathway for individuals from Cohort 1 was Pathway 3, and the least common was Pathway 4 . The percentages of euphausiids following each pathway were similar between Cohorts 2 and 3, that is, very few individuals from either of these cohorts followed Pathways 3 or 4 : the majority followed either Pathway 1 or Pathway 2. We only had complete molt records for 3 individuals from Cohort 4. Although all 3 of these followed Pathway 1 , other individuals from this cohort that survived through the FVII stage followed Pathway 2. contrast, Ross (1981) only found hatching percentages that high for $38 \%$ of the broods in her study. Iguchi \& Ikeda (1994) suggested that the higher hatching success in their study compared to that of Ross (1981) was due either to differences in experimental procedures or geographical variation in the sensitivity of eggs. The hatching success of the 4 broods used in our study ranged from 50 to $94 \%$, and overall our egg-hatching results from other experiments have varied widely (Gómez-Gutiérrez 2002). Perhaps this lends credibility to Iguchi \& Ikeda's (1994) hypothesis of a greater sensitivity of eggs in the NE Pacific, or possibly this variability is a function of the far greater sample sizes examined by Ross (1981) and ourselves than by Iguchi \& Ikeda 1994. (Ross: >3000; our study: >1000; Iguchi \& Ikeda: $~ 300$ eggs). In either case, both studies in the NE Pacific suggest that the failure of eggs to hatch could contribute significantly to the loss of Euphausia pacifica production.

Ross (1981) reported a survival rate of $2.4 \%$ from the $\mathrm{N} 1$ to juvenile stage at $8^{\circ} \mathrm{C}$ and $15.8 \%$ at $12^{\circ} \mathrm{C}$. Our survival rates from the $\mathrm{N} 1$ to juvenile stage at $10.5^{\circ} \mathrm{C}$ ranged from 6 to $15.2 \%$ for the various cohorts, consistent with her results. Ross (1981) also commented that the molt from metanauplius to $\mathrm{C} 1$ stage and the interval between stages $\mathrm{C} 1$ and $\mathrm{C} 3$ were periods of high mortality, resulting in a combined mortality of $>50 \%$ from N1 to C1. Iguchi \& Ikeda (1994) found that mortality was $34.4 \%$ from Stages $\mathrm{N} 1$ to $\mathrm{C} 1$ at $10^{\circ} \mathrm{C}$. These findings are also consistent with our results.

All females in this study were collected from the same plankton tow, minimizing the chances of differing feeding history, and all eggs and larvae were maintained under identical conditions. Despite this, we

\section{DISCUSSION}

The main goals of this study were to determine time to stage, stage duration and developmental pathways for larval Euphausia pacifica off the coast of Oregon, USA. We discuss the applicability of former studies to the population in our study area, the implication of our results for interpretation of field data, and the possible ecological implications of the high degree of developmental variability expressed by this species.

\section{Hatching and survival}

Our recorded hatching time of $39 \mathrm{~h}$ falls within the $2 \mathrm{~d}$ time period found by Iguchi \& Ikeda (1994) at $10^{\circ} \mathrm{C}$. Their mean hatching percentage for this temperature was $97 \%$; in

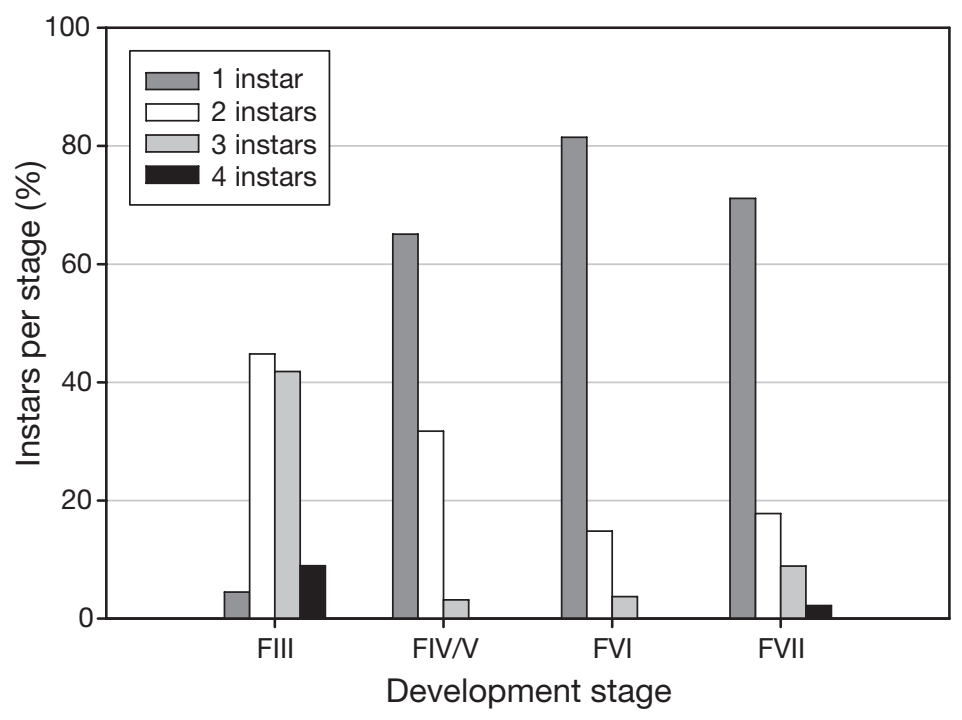

Fig. 6. Euphausia pacifica. Percentage of single and multiple instars at each development stage 
found considerable differences in both the percentage of eggs that hatched and in the survival to juveniles among the 4 cohorts. For example, Cohort 4 had substantially lower hatching and survival rates than the other 3 cohorts. Perhaps lower hatching success is indicative of embryos that were not endowed with sufficient nutritional reserves to assure hatching of a healthy nauplius. Ross (1981) proposed that egg size might be a determinant of egg hatching-success. We were unable to test this because, to minimize potential damage due to handling, we chose not to measure the eggs prior to hatching. The only other difference among cohorts was that the female that released the eggs for Cohort 4 produced fewer eggs and was smaller than the females associated with Cohorts 2 and 3 ; however she produced more eggs and was about the same size as the female from Cohort 1 (Table 1).

\section{Larval development}

The FV stage is rare in our study area, in contrast with southern California where this stage is commonly found (Boden 1950, Knight 1984, Rumsey 1999). FIV and FV were treated as a single stage in our study and we observed that $65 \%$ of individuals in FIV/V had only 1 instar, which means that they could not have passed through 2 stages. This supports our observation from preserved field samples that FV is often skipped by euphausiids in this area. This was also observed by Ross (1981) in Puget Sound. In Ross' (1981) study, 20\% of the euphausiids had 2 instars between Stages FIV and FVI, consistent with $31 \%$ in our study. Multiple

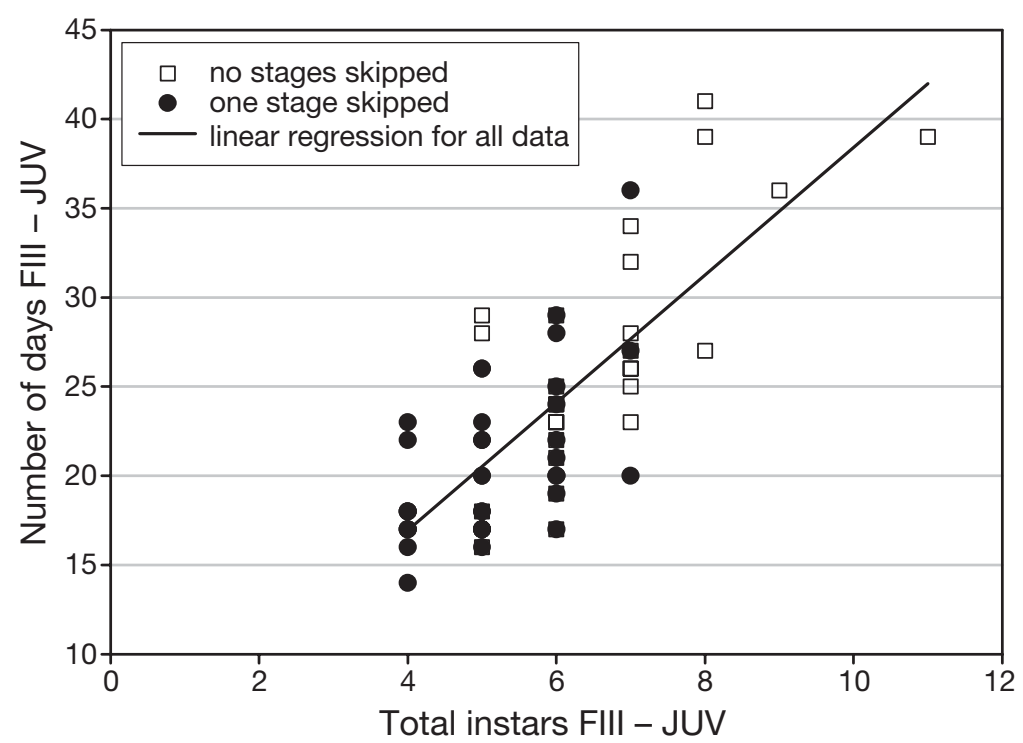

Fig. 7. Euphausia pacifica. Total number of instars versus period (d) between FIII and juvenile stages, comparing individuals that skipped stages with those that did not. Overall, $y=3.58 x+2.64, \mathrm{r}^{2}=0.5425$
Table 3. Euphausia pacifica. Percentage that followed each developmental pathway in each cohort (Pathway 1: no stages skipped; Pathways 2 to 4: 1 stage skipped - see Fig. 5). Only data for individuals that had complete molt records are included; n: total no. of euphausiids from each cohort that survived to juvenile

\begin{tabular}{|c|c|c|c|c|}
\hline \multirow[t]{2}{*}{ Pathway } & \multicolumn{4}{|c|}{ Cohort } \\
\hline & 1 & 2 & 3 & 4 \\
\hline 1 & 25 & 40 & 48 & 100 \\
\hline 2 & 25 & 40 & 35 & 0 \\
\hline 3 & 38 & 16 & 13 & 0 \\
\hline 4 & 12 & 4 & 4 & 0 \\
\hline $\mathrm{n}$ & 16 & 25 & 23 & 3 \\
\hline
\end{tabular}

instars do not necessarily indicate that all those euphausiids passed through FV, since other single stages commonly had multiple instars and this would probably be the case for FIV also (Fig. 6). Combining stages FIV and FV into a single stage was done for practical purposes in this study, but observations of NE Pacific populations indicate that this should not have greatly affected the results.

The range in stage was approximately $2 \mathrm{wk}$ for calyptopes, 3 wk for Stages FI and FII, and $>1$ mo for Stages FIII to FVII (Table 2). This means that larvae identified in field samples can often only be aged within \pm 1 mo. Bearing in mind that this range in development is derived from data on 3 batches of eggs produced on $1 \mathrm{~d}$, such a great range could make it difficult to track cohorts in the field, where much more variability would be expected. For example, if there were a strong pulse in eggs produced over a period of only a few days in the sea, the first individual FIII would be seen as early as $22 \mathrm{~d}$ later, but there could still be individuals in this stage until at least Day 58, 2 $\frac{1}{2}$ wk after the first individual had reached the juvenile stage (Table 2). We know that there are several peaks in egg production throughout the Euphausia pacifica spawning season off Oregon (Feinberg \& Peterson 2003). Thus, 'peaks' in abundance of later furcilia stages are likely to be smoothed to such a degree that stagebased cohorts would be completely obscured.

\section{Development patterns}

Because an extended period of development was observed for Stage C1, it appears that this stage may represent a bottleneck in the development of Euphausia pacifica larvae. There are physiological and behavioral reasons that 
may account for this. The transition to $\mathrm{C} 1$ is the first molt (nauplii do not molt, rather they metamorphose) and $\mathrm{C} 1$ is the first feeding stage for this species (Mauchline \& Fisher 1969). It is the stage at which a simple gut (Suh et al. 1994) and extensive musculature (Boden 1950) first develop. The extended duration of this stage may reflect a need to restore lipid reserves lost during metabolism in the 3 previous non-feeding stages. In addition, this stage may be protracted, since the molt from metanauplius to $\mathrm{C} 1$ results in a highly altered morphology. The first feeding stage of euphausiids is very sensitive to the physical and feeding environments (Brinton 1976, Ikeda 1984, Ross et al. 1988, Ross \& Quetin 1989, Paul et al. 1990). In the field, it would be critical for E. pacifica larvae to reach C1 in an environment of plentiful food. The differential ability of individuals to complete this transition from metanauplius to $\mathrm{C} 1$ may explain why the range in development times increased significantly at this stage. Similar patterns have been reported for copepods (Calanus spp. Landry 1983, Peterson 1986, 2001, Uye 1988, Peterson \& Painting 1990), in which the third nauplius has prolonged development.

Furcilia III may also be a developmental bottleneck for Euphausia pacifica. It is the longest-lasting stage, and the first stage at which all 5 pleopods are setose and more advanced gill and eye development are evident (Boden 1950). There is also evidence in the literature (Iguchi 1995) and from our field data that by Stage FIII vertical migration behavior has become well established. This combination of physiological and behavioral changes marks the point in development when the larvae begin to resemble adult euphausiids. The larvae in our experiments never skipped the FIII stage, and 1, 2 and 3 instars within this stage were common (Fig. 6). Ross (1981) reported similar results, although a single instar in this stage was far less common in her study. Boden (1950) found that FIII was the most abundant stage in his samples collected off Southern California, as did we (unpubl. data), consistent with a development stage of extended duration. The frequency of multiple instars at FIII suggests that some individuals required more time at this stage. This could result from complex developmental changes and/or a physiological size threshold that must be attained before progressing to the next stage. Ross (1981) found that larvae which grew significantly between Stages FIII and FIV/V were less likely to have multiple instars between Stages FIV/V and FVI than larvae that did not grow significantly between these stages. Such analysis was not possible for the transition from FII to FIII, but we assume that the same principle could apply.

Certain aspects of the development patterns of Euphausia pacifica discussed above are clearly con- trolled by physiological constraints, such as prolonged development at the C1 and FIII stages. We speculate that the early development of euphausiids (embryo to C1) is also a result of evolutionary constraints. Given that the embryos hatch quickly and that they can pass through 3 stages within $5 \mathrm{~d}$ before reaching the first feeding stage (C1), we suggest that natural mortality in these early stages is very high (half of our experimental euphausiids had died by this stage), and that there has been selection for a swimming nauplius to hatch out of the embryo as quickly as possible, with subsequent development of a gut and the feeding apparatus dependent upon lipid reserves. The development of these structures outside the embryo might have evolved because of high egg-mortality rates.

\section{Developmental variability}

Of the 3 cohorts with highest survival, Cohort 1 reached the juvenile stage 7 to $9 \mathrm{~d}$ faster than Cohorts 2 or 3 . Ross (1981) also found significantly different times to the juvenile stage among the cohorts in her study. These 'times to juvenile' reflect the median time for a cohort, but the differences between cohorts can be partially explained by the variable developmental pathways followed by individuals within the cohorts. We found that $75 \%$ of the euphausiids in Cohort 1 skipped 1 of the late furcilia stages, whereas only 40 to $50 \%$ of Cohorts 2 and 3 did so (Table 3 ).

The highly variable number of instars per stage and the frequency of skipped stages was especially surprising given that the euphausiids were raised under identical environmental conditions. Over $50 \%$ of the individuals in our experiment skipped a development stage between FIII and juvenile, compared to 5\% reported by Ross (1981). Gopalakrishnan (1973) did not see any evidence that Nematoscelis difficilis skipped development stages. $N$. difficilis is a less widely distributed species than Euphausia pacifica, and perhaps its distribution is limited by its relative inflexibility in development. $N$. difficilis is also more oceanic in distribution, and several authors have suggested that oceanic species are more likely to display stable developmental sequences than species which inhabit more diverse neritic regions (summarized by GómezGuttiérez 1996).

The increase in frequency of skipped stages between our study and that of Ross (1981) may be attributable to differences in development between oceanic and an isolated Puget Sound population. Alternatively, differences in experimental diets may be partially responsible. Our euphausiids were fed a mixture of 4 different algae, compared to the single Thalassiosira spp. diet of Ross (1981). Le Roux's $(1973,1974)$ studies 
on the euphausiids Nyctiphanes couchii and Meganyctiphanes norvegica found that individuals fed unialgal diets required additional time to complete larval development. The multi-algal diet in our experiment may have provided superior nutritional resources and contributed to the higher frequency of skipped stages.

Although we found a much higher incidence of skipped stages than did Ross (1981), the total development time to juveniles was comparable to that in her study. This may be attributable to the common occurrence of multiple instars per stage in our euphausiids. The incidence of multiple instars in Stages FIII to FVI was similar in both studies. Single instars at FVII were far more common in our study $(70 \%$, compared to $48 \%$ in Ross' 1981 study). Both studies documented a multitude of developmental sequences with varying degrees of directness. The most direct (fastest) routes were those in which the euphausiids had the fewest total instars. Overall, the individuals in our study had from 4 to 11 instars between the FIII and juvenile stages, with an average of 6 instars (Fig. 7). Individuals that skipped 1 stage had an average of 5 instars and those that did not had an average of 7 instars. Our interpretation of Ross' (1981) data is that the euphausiids in her study ranged from 4 to 9 instars between the FIII and juvenile stages, with an average of 6 instars. The variability in possible developmental pathways was higher in our study, despite the fact that the average number of instars in both studies was the same.

Siblings showed no tendency to follow the same developmental pathway (Table 3). The overall variability in developmental pathways within a cohort indicates that adults do not confer a particular pattern of development on their offspring although, within a cohort, some pathways tend to be followed more frequently than others (Table 3). Cohorts 2 and 3 had similar percentages of individuals following each pathway, and these cohorts had similar median development times. Pathway 1 (no stages skipped) was a dominant pathway for all cohorts except Cohort 1 , and consequently this cohort had the fastest median time to the juvenile stage (Fig. 4). These results suggest that a degree of variability is inherent to a cohort, allowing flexibility in development for the whole brood.

Our median times to stage are within the range of Euphausia pacifica development times found by Ross (1981). Thus we assume that her rates, as well as our own, can be applied to field data from varying locations within the range of 8 to $12^{\circ} \mathrm{C}$. Iguchi \& Ikeda (1994) followed development only until the $\mathrm{C} 1$ stage, and their result of $6.2 \mathrm{~d}$ for $10^{\circ} \mathrm{C}$ is consistent with our median development time of $6.4 \mathrm{~d}$ to $\mathrm{C} 1$. Unfortunately, they did not continue their experiments through the furcilia stages, so we have no opportunity for comparison with populations in the western Pacific.

Although there is clearly inherent variability in the developmental pathways of individuals, temperature may also have an effect on development and number of instars per stage. Neither our study nor that of Ross (1981) could address variability in the developmental pathway for the first 2 furcilia stages (which is often recorded in field studies), although we would expect the same relationship with temperature and directness of development to persist through later development stages. Our $10.5^{\circ} \mathrm{C}$ data and the $8^{\circ} \mathrm{C}$ data of Ross (1981) showed a deceleration in development starting at FIII, but at $12^{\circ} \mathrm{C}$ no deceleration was observed (Fig. 8). Rumsey (1999) also found that indirect (slower) developmental pathways for Euphausia pacifica were only associated with his 'cool' stations, although this may have been mitigated by anomalously high chlorophyll values at all stations in his study. Other studies of euphausiids in the eastern Pacific (Knight 1984, Lavaniegos 1994, Gómez-Gutiérrez 1996) show the opposite trend from that suggested by our Fig. 8. Slower (less direct) development was associated with an increase in number of instars in the FI and FII stages at warmer temperatures; however these temperatures were always associated with lower chlorophyll levels. The median values in Fig. 8 only show the end result in development time to a stage, not the number of instars required to reach it. Differences in development patterns with temperature could have

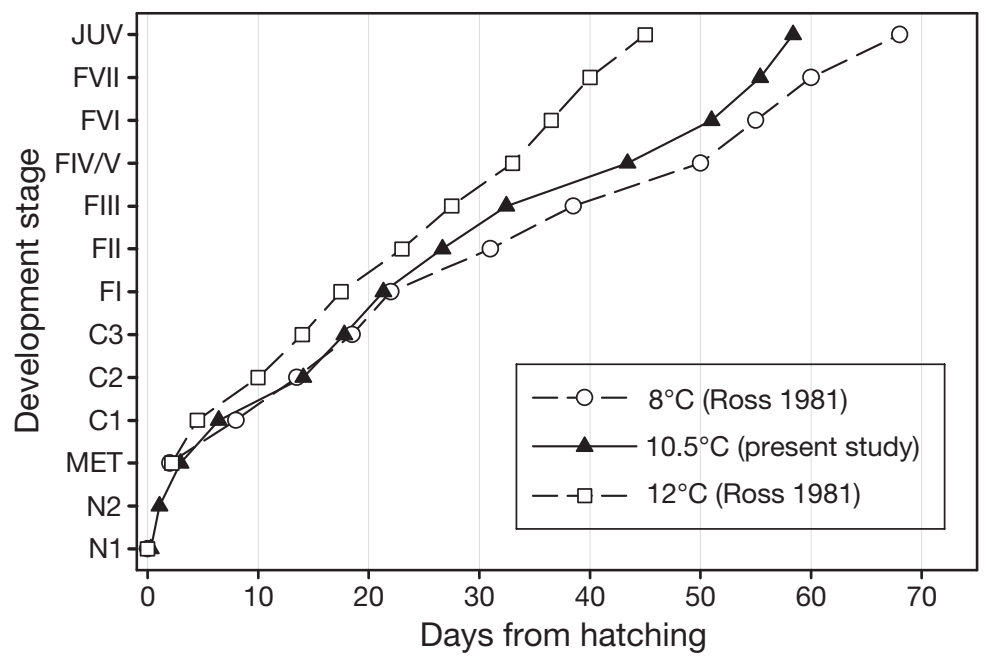

Fig. 8. Euphausia pacifica. Median time to development stages at 3 temperatures. Data for 8 and $12^{\circ} \mathrm{C}$ represent medians from all experiments at those temperatures (Ross 1981). $10.5^{\circ} \mathrm{C}$ data represent median for Cohorts 1 to 3 of current study 
been caused by a temperature-related change in intermolt period, an increase in the number of instars within individual furcilia stages, and/or a decrease in the number of skipped stages (Fig. 8). Since food levels stayed constant regardless of temperature in both our study and that of Ross (1981), we suggest that temperature and not just food availability may have an overall impact on the larval development of the euphausiids.

Metabolically, faster development at higher temperatures is expected, but this assumption may not be valid for upwelling systems. In an upwelling region, sustained warmer temperatures are often associated with periods of poorer productivity, and thus lower food availability for the euphausiids. Lower temperatures in the field off the coast of Oregon might result in less developmental deceleration than seen in our experiments due to the compensatory effect of an increased food supply during upwelling.

Brinton (1962, 1976) suggested that Euphausia pacifica larvae are well-adapted to seasonal and interannual variability in physical and biological oceanographic conditions. Variability in developmental rates in a dynamic coastal environment such as the Oregon upwelling region may increase the chance that some individuals in a brood will survive. Developing at different rates increases the likelihood that some individuals from the brood will be at the right stage to take advantage of favorable environmental conditions when they occur. Rapid development to the juvenile stage may confer a survival advantage, as juveniles are active swimmers and are better able to find food and avoid predation than the smaller, less mobile larval stages. Rapid development would be particularly advantageous for individuals hatched in spring, as they could potentially reproduce during that same year.

We hypothesize that slower development may also be advantageous under certain conditions. The lower productivity during poor upwelling seasons may favor individuals with slower development and therefore lower energy requirements. Slower development could be reproductively advantageous to individuals hatched in late summer. The main pulse of egg production off the Oregon coast occurs in late summer (Feinberg \& Peterson 2003). Even the fastest-developing individuals hatched at this time of year would be unlikely to reach maturity and be ready to reproduce before winter (when food resources are substantially decreased), minimizing the potential reproductive advantage of rapid development at this time of year. Individuals that develop more slowly may eventually reach the adult stage at a larger size, which could be advantageous for overwintering and, ultimately, for reproduction the following year.

\section{CONCLUSIONS}

The developmental rates we recorded were within the range of those in other studies, indicating that at a given temperature development rates may be comparable among populations. However, our study showed a higher incidence of single instars and skipped stages in the later furcilia stages than shown by previous studies. The slower-developing stages were C1 and FIII. Several physiological constraints occur during these stages, suggesting that these 2 stages represent bottlenecks in development. There was variability in time to stage and development pattern within and among cohorts, suggesting that variability is intrinsic to the individual. This flexibility probably allows Euphausia pacifica to thrive, and often dominate, in both dynamic shelf regions and more constant oceanic conditions. In this study, the most significant differences observed among cohorts and among different experimental temperatures occurred in the late furcilia stages. More than half of the larvae in our experiments skipped one of the later furcilia stages. This, combined with the $>1$ mo range in stage of later furcilia, should be considered when using field data for cohort analysis or to determine mortality rates.

Acknowledgements. We are grateful to J. Gómez-Gutiérrez, J. Lamb, J. Keister, M. Vance and C. Ryan for assistance with these experiments. This work would have been far more difficult without the algae cultured and provided by E. Onal and the Oregon State University Molluscan Broodstock Program. We thank the US GLOBEC program for funding this work and C. Morgan for helpful commentary on the manuscript. This is publication number 300 from the NE Pacific US GLOBEC program.

\section{LITERATURE CITED}

Boden BP (1950) The post-naupliar stages of the crustacean Euphausia pacifica. Trans Am Microsc Soc 69:373-386

Bollens SM, Frost BW, Lin TS (1992) Recruitment, growth and diel vertical migration of Euphausia pacifica in a temperate fjord. Mar Biol 114:219-228

Brinton E (1962) The distribution of Pacific euphausiids. Bull Scripps Inst Oceanogr Univ Calif 8:51-269

Brinton E (1976) Population biology of Euphausia pacifica off southern California. US Fish Wildl Serv Fish Bull 74:733-762

Feinberg LR, Peterson WT (2003) Variability in duration and intensity of euphausiid spawning off central Oregon, 1996-2001. Prog Oceanogr 57:363-379

Gómez-Gutiérrez J (1996) Ecology of early larval development of Nyctiphanes simplex Hansen (Euphausiacea) off the southwest coast of Baja California, Mexico. Bull Mar Sci 58:131-146

Gómez-Gutiérrez J (2002) Hatching mechanism and delayed hatching of the eggs of three broadcast euphausiid species under laboratory conditions. J Plankton Res 24:1265-1276

Gopalakrishnan K (1973) Developmental and growth studies of the euphausiid Nematoscelis difficilis (Crustacea) based on rearing. Bull Scripps Inst Oceanogr Univ Calif 20:1-87 
Iguchi N (1995) Spring diel migration of a euphausiid Euphausia pacifica in Toyama Bay, Southern Japan Sea. Bull Jpn Sea Natl Fish Res Inst 45:59-68

Iguchi N, Ikeda T (1994) Experimental study on brood size, egg hatchability, and early development of a euphausiid Euphausia pacifica from Toyama Bay, Southern Japan Sea. Bull Jpn Sea Natl Fish Res Inst 44:49-57

Iguchi N, Ikeda T, Imamura A (1993) Growth and life cycle of a euphausiid crustacean (Euphausia pacifica Hansen) in Toyama Bay, Southern Japan Sea. Bull Jpn Sea Natl Fish Res Inst 43:69-81

Ikeda T (1984) Development of the larvae of the Antarctic krill Euphausia superba observed in the laboratory. J Exp Mar Biol Ecol 75:107-117

Knight MD (1984) Variation in larval morphogenesis within the southern California Bight population of Euphausia pacifica from winter through summer, 1977-1978 Calif Coop Ocean Fish Investig Rep 25:87-99

Landry MR (1983) The development of marine calanoid copepods with comment on the isochronal rule. Limnol Oceanogr 28:614-624

Lavaniegos BE (1994) Dispersion and development patterns in larvae of Nyctiphanes simplex (Euphausiacea) in the upwelling region off Baja, California. Mar Ecol Prog Ser 106:207-225

Le Roux A (1973) Obsérvations sur le developpement larvaire de Nyctiphanes couchii (Crustacea: Euphausiacea) au laboratoire. Mar Biol 22:159-166

Le Roux A (1974) Obsérvations sur le developpement larvaire de Meganyctiphanes norvegica (Crustacea: Euphausiacea) au laboratoire. Mar Biol 26:45-56

Matthiessen GC, Toner RC (1966) Possible methods of improving the shellfish industry of Martha's Vineyard, Duke's County, Massachusetts. Marine Research Foundation, Edgartown, MA

Mauchline J, Fisher LR (1969) The biology of euphausiids. Adv Mar Biol 7:1-454

Paul AJ, Coyle KO, Ziemann DA (1990) Timing of spawning of Thysanoessa raschii (Euphausiacea) and occurrence of their feeding stage larvae in an Alaskan Bay. J Crustac Biol 10:69-78

Peterson WT (1986) Development, growth and survivorship of the copepod Calanus marshallae in the laboratory. Mar Ecol Prog Ser 29: 61-72

Peterson WT (2001) Patterns in stage duration and development among marine and freshwater calanoid and cyclopoid copepods: a review of rules, physiological constraints, and evolutionary significance. Hydrobiologia 453/454:91-105

Editorial responsibility: Otto Kinne (Editor-in-Chief), Oldendorf/Luhe, Germany
Peterson WT, Painting SJ (1990) Development rates of the copepods Calanus australis and Calanoides carinatus in the laboratory, with discussion of methods used for calculation of development times. J Plankton Res 12:283-293

Ponomareva LA (1966) Euphausiids of the North Pacific, their distribution and ecology. (Transl. of 1963 paper) Israel Program of Scientific Translations, Jerusalem

Ross RM (1981) Laboratory culture and development of Euphausia pacifica. Limnol Oceanogr 2:235-246

Ross RM, Quetin LB (1989) Energetic cost to develop to the first feeding stage of Euphausia superba Dana and the effects of delays in food availability. J Exp Mar Biol Ecol 133:103-127

Ross RM, Daly KL, English TS (1982) Reproductive cycle and fecundity of Euphausia pacifica in Puget Sound, Washington. Limnol Oceanogr 27:304-314

Ross RM, Quetin, LB, Kirsch E (1988) Effect of temperature on developmental times and survival of early larval stages of Euphausia superba Dana. J Exp Mar Biol Ecol 121:55-71

Rumsey SM (1999) Environmentally forced variability in larval development of Euphausia pacifica: implications on larval survival and recruitment success in the southern California Bight. PhD dissertation, University of California, San Diego, CA

Siegel V, Nicol S (2000) Population parameters. In: Iverson I (ed) Krill biology, ecology and fisheries. Blackwell Scientific Publications, Oxford, p 103-149

Smiles MC, Pearcy WG (1971) Size, structure and growth of Euphausia pacifica off the Oregon coast. US Fish Wildl Serv Fish Bull 69:79-86

Sokal RR, Rohlf FJ (1969) Biometry. The principles and practice of statistics in biological research. WH Freeman \& Co, San Francisco, CA

Suh HL, Soh HY, Hong SY (1993) Larval development of the euphausiid Euphausia pacifica in the Yellow Sea. Mar Biol 115:625-633

Suh HL, Toda T, Hong SY (1994) Ontogeny of foregut morphology in the euphausiid Euphausia pacifica. J Crustac Biol 14:47-53

Tanasichuk RW (1998) Interannual variations in the population biology and productivity of the euphausiid Euphausia pacifica in Barkley Sound, Canada, with special reference to the 1992 and 1993 warm ocean years. Mar Ecol Prog Ser 173:163-180

Uye SI (1988) Temperature-dependent development and growth of Calanus sinicus (Copepoda; Calanoida) in the laboratory. Hydrobiologia 167/168:285-293

Zar JH (1999) Biostatistical analysis, 4th edn. Prentice-Hall, Upper Saddle River, NJ

Submitted: March 4, 2005; Accepted: December 1, 2005

Proofs received from author(s): June 13, 2006 